REVIEW

\title{
Carotid and vertebral artery dissection syndromes
}

\author{
B Thanvi, S K Munshi, S L Dawson, T G Robinson
}

Postgrad Med J 2005;81:383-388. doi: 10.1136/pgmj.2003.016774

Cervicocerebral arterial dissections (CAD) are an important cause of strokes in younger patients accounting for nearly $20 \%$ of strokes in patients under the age of 45 years. Extracranial internal carotid artery dissections comprise $70 \%-80 \%$ and extracranial vertebral dissections account for about $15 \%$ of all CAD. Aetiopathogenesis of CAD is incompletely understood, though trauma, respiratory infections, and underlying arteriopathy are considered important. A typical picture of local pain, headache, and ipsilateral Horner's syndrome followed after several hours by cerebral or retinal ischaemia is rare. Doppler ultrasound, MRI/MRA, and CT angiography are useful non-invasive diagnostic tests. The treatment of extracranial $C A D$ is mainly medical using anticoagulants or antiplatelet agents although controlled studies to show their effectiveness are lacking. The prognosis of extracranial CAD is generally much better than that of the intracranial CAD. Recurrences are rare in CAD.

See end of article for authors' affiliations

Correspondence to: Dr B Thanvi, Department of Integrated Medicine, Leicester General Hospital, Gwendolen Road, University Hospitals of Leicester NHS Trust, Leicester LE5 4PW, UK; bthanvi@hotmail.com

Submitted

6 November 2003

Accepted 31 August 2004
$\mathrm{T}$ he term dissection implies a tear in the wall of a major artery leading to the intrusion of blood within the layers of an arterial wall (intramural haematoma). This causes stenosis of the lumen when blood collects between the intima and media or an aneurysmal dilatation of the artery when the haematoma predominantly involves the media and adventitia. Once thought to be rare, cervicocerebral arterial dissections (CADs) have been increasingly recognised as a cause of stroke, particularly in young people. It was the work of Fisher et al in the late 1970s that led to the recognition of the clinical and radiological features of dissection syndromes CADs, internal carotid artery dissections (ICDs) are much more common than vertebral dissections. The aetiopathogenesis of CAD is not fully understood. A history of preceding neck trauma is common although not universal. Heritable connective disorders such as Ehlers-Danlos syndrome, Marfan's syndrome, $\alpha_{1}$ antitrypsin deficiency, etc, may predispose to CAD.

Typical clinical features of CAD include unilateral neck pain, headache, ipsilateral Horner's syndrome followed by manifestations of the cerebral or ocular ischaemia and cranial nerve palsies. The CAD can cause neurological deficits either because of haemodynamic failure (caused by luminal stenosis) or by an artery to artery thromboembolism. Catheter angiography had been the method of choice to diagnose arterial facilitating their antemortem diagnosis. ${ }^{1}$ Among dissections, but with the advent of Doppler ultrasonography, MRI/MRA, and CT angiography, most dissections can now be diagnosed non-invasively. The treatment of symptomatic CAD is essentially medical and neurosurgical interventions are rarely required. As thromboembolism is the most probable mechanism behind any neurological deficit, anticoagulants (heparin and warfarin) and antiplatetelets are commonly used, although there have not been any randomised studies to evaluate their effectiveness. The prognosis depends on the severity of neurological deficit but is generally good in extracranial dissections. The recurrence rate of CAD is very low. This review focuses on the pathogenesis, clinical features, diagnosis, and medical treatment of these syndromes.

\section{EPIDEMIOLOGY}

Although reported in all age groups, CADs are most common between the ages of 35 and 50 years, with a peak incidence in the fifth decade. ${ }^{2}$ They account for up to $20 \%$ of strokes in patients under the age of 45 years. ${ }^{3}$ A community based study showed an incidence of cervicocerebral dissections of 2.6 per 100000 per year. ${ }^{4}$ However, cervicocerebral dissections may be asymptomatic or may cause only minor symptoms and therefore, their true incidence is difficult to ascertain. The incidence of CADs is non-significantly higher in women. Trauma during forceps delivery can cause CAD in neonates.

\section{CLASSIFICATION OF CERVICOCEREBRAL DISSECTION SYNDROMES}

CADs include ICDs and vertebral artery dissections (VADs). In each arterial system, the segment of the artery involved can be extracranial (dissection involving the artery in the neck before it enters the cranium) or intracranial (when dissections affect the intracranial part of the artery) (box). ICD are at least three times commoner than VAD. The clinical features in these two types are distinct in view of the different areas of brain supplied by the two arteries. Dissections tend to affect extracranial segments of the arteries much more commonly than intracranial segments. However, intracranial dissections are usually more severe and carry a poorer prognosis than extracranial dissections. The increased incidence of extracranial involvement has been explained by the fact that this segment of the artery is more mobile and is also

Abbreviations: $C A D$, cervicocerbral arterial dissection; $I C D$, internal carotid dissection; $V A D$, vertebral artery dissection; EICD, extracranial internal carotid artery dissection 
prone to damage by bony structures such as the vertebrae and styloid processes. ${ }^{5}$

The site predilection for CAD is quite different from that of atherosclerosis affecting the cervical arteries. Dissection involves the pharyngeal and distal parts of the internal carotid artery, whereas atherosclerosis usually affects the origin and the carotid bulb. Similarly, dissections affect distal parts of the extracranial vertebral artery, whereas atherosclerosis tends to involve the proximal segments

\section{AETIOPATHOGENESIS OF CAD}

The pathogenesis of CAD is not fully understood. The following discussion outlines the current views on this subject.

\section{Where does the dissection start in the artery?}

An arterial wall consists of three layers-intima (the innermost layer), media (the middle muscular layer), and adventitia (the outermost layer). It is generally agreed that a tear in the wall of an artery leads to a collection of blood between the layers of the artery, leading to formation of an intramural haematoma. However, there is no universal agreement as to which wall is the primary site of dissection. Some authorities consider a rupture within the connective tissue and vasa vasorum of the media as the most probable initial event in dissection. ${ }^{6}$ The intramural haematoma may then later penetrate the intima and reconnect with the true arterial lumen. Others think that an intimal tear occurs, ${ }^{7}$ which allows blood under arterial pressure to enter the wall of the artery.

Intimal tears are not always easy to diagnose on pathological specimens. Even in the carefully selected patients it is not always possible to show a communication between the intramural haematoma (the so called false lumen) and the true lumen, suggesting that some dissections of carotid and vertebral arteries may be caused by a primary intramural haematoma.

\section{What are the causes/triggers of a CAD?}

Several factors have been proposed as causative or trigger factors for arterial dissections.

\section{Trauma}

Cervicocerebral dissections are often preceded by some form of trauma to the neck. However, the severity of trauma can range from a trivial injury (for example, vigorous nose blowing) to severe roadside accidents involving direct injury to the neck arteries. Practically all forms of neck trauma have been reported to be associated with CAD including chiropractic manoeuvres, vigorous coughing or nose blowing, sudden neck turning as in reversing a car, prolonged telephone conversations, road accidents inflicting direct trauma to a cervical artery, strangulations, bar fights, recent surgery involving prolonged anaesthesia, etc.

It is conceivable that trauma leads to a tear in the intima or media. However, it is difficult to explain how minor neck

\section{Common types of cervicocerebral dissections}

- Internal carotid artery dissection

- extracranial dissections

- intracranial dissections

- Vertebral artery dissections

- extracranial dissections

- intracranial dissections trauma can cause dissection of the arteries and in many cases there is no clear history of antecedent trauma in some cases.

\section{Genetic factors}

Several inherited disorders of connective tissue are known to be associated with CAD although in practice they are rarely found. Most typical of these is vascular Ehler-Danlos syndrome (EDS), an autosomal dominant disorder. Patients with this disorder often carry mutations in the gene that encodes pro- $\alpha$ l (III) collagen. ${ }^{8}$ Brandt et al showed ultrastructural abnormalities of dermal connective tissue components in two thirds of patients with spontaneous dissection of the carotid or vertebral artery but with no other stigmata of a known connective tissue disorder. ' CADs have also been reported in association with fibromuscular dysplasia, a noninflammatory disease of medium sized arteries mainly affecting the carotid and renal arteries. ${ }^{10}$ Patients with ICD are more likely to have fibromuscular dysplasia than patients with VAD. ${ }^{11}$

$\alpha_{1}$ Antitrypsin deficiency ${ }^{12}$ and abnormalities of neural crest cells, such as angiolipomatosis and lentigenosis ${ }^{13}{ }^{14}$ have also been associated with an increased risk of CAD. In about $5 \%$ of patients with CAD, there is a family history of spontaneous arterial dissections. ${ }^{15}$ To date no mutations in the gene for type III procollagen (COL3AI) have been identified. ${ }^{16}$ The CAD has also been reported in patients with osteogenesis imperfecta. ${ }^{17}$

Interestingly, hyperhomocysteinaemia has recently been reported to be an in association with $\mathrm{CAD}^{18}$; Pezzini et al report higher levels of fasting total plasma homocysteine (tHcy) in patients with spontaneous CAD (13.2 $\mu \mathrm{mol} / \mathrm{l}$; range, 7 to $32.8 \mu \mathrm{mol} / \mathrm{l}$ ) compared with control subjects $(8.9 \mu \mathrm{mol} / \mathrm{l}$; range, 5 to $17.3 \mu \mathrm{mol} / \mathrm{l}) .{ }^{19}$ The authors also noted a significant association between the MTHFRT (methylenetetrahydrofolate reductase) genotype and spontaneous CAD. A mutation in the coding region of MTHFRT is associated with a variant form of the enzyme that has a reduced level of activity. The authors suggest several possible biological mechanisms to explain the relation between the increased plasma homocysteine concentrations and arterial dissections. These include increased elastolytic activity and inhibition of the cross linking of collagen caused by hyperhomocysteinaemia.The detailed account of the biochemical mechanisms underlying association of hyperhomocysteinaemia and CAD is beyond the scope of this article and the reader is referred to a recent article by Rosner on this subject. ${ }^{20}$

The occasional association of spontaneous dissections with intracranial aneurysms $s^{21}$ and arterial redundancies (for example, coils, kinks, and loops) also supports the theory of an underlying arteriopathy. ${ }^{22}$ Recently, some authors have reported an evidence of impaired endothelium dependent vasodilatation in CAD patients that is not the result of stroke, and they suggest that an underlying abnormality of the arterial wall layers may predispose to $\mathrm{CAD} .{ }^{23}$ However, the theory of an underlying arteriopathy fails to explain the specific site predilection of dissections, their low recurrence rate, and the rarity of a family history in CAD. It is possible that in the familial cases, the penetrance of the genetic predisposition is low.

\section{Infections}

A recent infection (particularly a respiratory infection) has been suggested by some authors to predispose to the CAD. ${ }^{24}$ In a case controlled study, ${ }^{25}$ an acute infection was more frequent in patients with the spontaneous CAD (31.9\%) than in control subjects (13.5\%) This association was stronger in patients with multiple (odds ratio, 6.4) rather than single artery (odds ratio, 2.1) dissection. The damage to the arterial 
wall may be caused by proteolytic, oxidative, or autoimmune mechanisms. This theory is also supported by the finding of seasonal variations in the incidence of CAD, with the highest incidence in the fall. ${ }^{26}$ However, no direct evidence exists for a specific infectious agent.

\section{Miscellaneous factors}

CAD has also been reported in associations with migraine, ${ }^{27}$ smoking, hypertension, and contraceptive use.

In summary, the pathogenesis of CAD presumably entails an interaction of genetic and environmental factors (figl).

What is the mechanism of neurological deficit(s)? Local thrombus formation is promoted by the luminal stenosis and the release of thrombogenic factors by the intimal damage. Cerebral ischaemia can be produced by (1) a narrowed lumen with the consequent haemadynamic failure or, (2) embolisation from local thrombus, or both. Embolisation seems to be the main mechanism as suggested by (1) the detection of distal emboli on angiography in patients with CAD, (2) the evidence of microemboli signals on Doppler ultrasound in many cases, and (3) evidence of cerebral ischaemia or infarct in the absence of a significant luminal stenosis, or both. This is further corroborated by a study $^{28}$ that found territorial infarcts indicative of thromboembolic disease in all 141 consecutive patients with spontaneous carotid dissections on CT, MRI, and ultrasound. Additional border zone infarcts, indicating haemodynamic infarction, were seen in six patients.

Horner's syndrome and cranial nerve palsies (particularly the lower nerves) are the result of local dissection effects.

\section{Clinical features of CAD}

The clinical presentation of CAD depends on the artery involved and the location of the dissection, for example, extracranial compared with intracranial. Typically, CAD present as pain, headache, Horner's syndrome, lower cranial nerve palsies, cerebral or ocular TIAs, and/or strokes. Clinical aspects of main cervicocerebral dissections are described below.

\section{EXTRACRANIAL INTERNAL CAROTID ARTERY DISSECTIONS (EICD)}

Cervical internal carotid dissections are the commonest type of CAD and account for nearly $2.5 \%$ of all first strokes. ${ }^{29}$ They occur most commonly between the ages of 35 and 50,

\section{Genetic factors}

Ehlers-Danlos syndrome

Fibromuscular dysplasia

Ultrastructural connective tissue abnormalities

Homocystinaemia

Marfan's syndrome, cystic medial necrosis

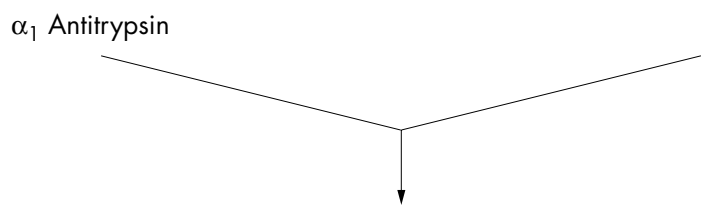

Cervicocerebral arterial dissections

Figure 1 Schema of interaction of genetic and environmental factors in the pathogenesis of cervicocerebral dissections. affecting both sexes equally. The classic triad of pain on one side of the head, face, or neck, partial Horner's syndrome followed hours or days later by cerebral or retinal ischaemia is found in less than one third of patients, but the presence of any two elements of this triad is strongly suggestive of the diagnosis. ${ }^{7}$ The clinical features of EICD can be divided into two groups depending on whether they result from the local effects of the dissection or from the distal ischaemia.

\section{Clinical manifestations attributable to local effects Pain}

Neck pain and headache are usually the most prominent features of the EICD and may be the only manifestations. They may precede cerebral or ocular ischaemic symptoms by hours or days whereas in atherosclerotic stroke headache usually accompanies or follows the onset of neurological deficit. This may provide an important differentiating clue from atherosclerotic aetiology. The pain is usually sharp and may affect the jaw or face. The headache is usually constant and non-throbbing, although severe throbbing headache, "thunderclap" headache, or gradually worsening headaches have also been reported. It mostly affects the frontal or frontoparietal area. ${ }^{29}$ Sometimes it can be confused with a migraine episode or a subarachnoid bleed..$^{30}$ About two thirds of the patients report an ipsilateral headache.

\section{Partial Horner's syndrome}

The sympathetic fibres that travel along the internal carotid artery can be affected in EICD causing Horner's syndrome on the ipsilateral side. The syndrome is usually partial and is characterised by ptosis and miosis. There is no anhidrosis as fibres for sweat function in the face travel along the external carotid artery. Nearly 50\% of EICD present with the Horner's syndrome. $^{31}$

Pulsatile tinnitus or a subjective bruit may be noted in about one third of patients.

Ipsilateral cranial nerve palsies can be a local manifestation of EICD. Any cranial nerve can be involved but lower nerves are more often implicated. Isolated palsies of the hypoglossal nerve and sixth nerve have been reported. Taste disturbance and tongue weakness are the most common manifestations and are the result of compression and ischaemia of the XII nerve because of its proximity to the carotid sheath. A combination of ipsilateral cranial nerve palsy and contralateral hemispheric deficit in EICD may mimic brain stem ischaemia and is termed a "false localising sign". ${ }^{32}$

\section{Clinical manifestations attributable to ischaemia}

EICD may cause ipsilateral cerebral or retinal ischaemia in $40 \%-90 \%$ of cases..$^{33}$ The ischaemic signs tend to follow local symptoms by hours to a few days. Because of the increased awareness among physicians of CAD coupled with the availability of non-invasive tests, it is probable that the dissections are now diagnosed early and in the absence of any neurological deficit.

The ischaemia may cause TIAs or infarctions, or both. Both cerebral and retinal ischaemic manifestations have been reported, including amaurosis fugax, hemiplegia, dysphasia, etc. The TIA periods are usually more frequent than in the atherosclerotic stroke, described by Fisher as carotid allegro. ${ }^{1}$ Cerebral infarctions are usually preceded by TIAs. Ischaemic optic neuropathy attributable to the occlusion of arteries supplying the optic nerve may rarely occur if the dissection extends to the carotid siphon. ${ }^{34}$

\section{INTRACRANIAL CAROTID SYSTEM DISSECTIONS}

It is important to differentiate extracranial dissection from intracranial dissection for several reasons: 
- Intracranial dissections are much less common than extracranial dissections. ${ }^{35}$ However, it is possible that because of the lack of specific angiographic features, many non-fatal intracranial dissections are not diagnosed.

- Patients with intracranial dissections are generally younger than those with extracranial dissections, most commonly in their second or third decades.

- Intracranial dissections are usually associated with large strokes. A mortality of $75 \%$ has been reported in these dissections.

- Subarachnoid haemorrhage (SAH) can result from intracranial dissections, because of the extension of an intramural haematoma through the adventitia.

- Intracranial dissections can cause aneurysmal dilatations of the arteries that may behave as space occupying lesions compressing adjacent cranial nerves or the brain.

- Surgical interventions are more often needed in intracranial dissections.

\section{DISSECTIONS OF VERTEBROBASILAR SYSTEM Extracranial vertebral dissections}

Neck trauma may clearly precede an extracranial vertebral dissection. The vertebral artery is most mobile and thus most vulnerable to mechanical injury at $\mathrm{Cl}$ to $\mathrm{C} 2$ as it leaves the transverse foramen of the axis vertebra and suddenly turns to enter the intracranial cavity. Women are 2.5 times more frequently affected by the extracranial vertebral dissections than men and the reverse is true for intracranial dissections. ${ }^{36}$ Clinical manifestations include severe neck pain mostly in the occipitocervical area followed after a variable interval by ischaemic symptoms. In some patients there may not be any ischaemic symptoms. Dizziness, vertigo, double vision, ataxia, and dysarthria are common clinical features. TIAs are less common than in ICA dissections. Lateral medullary (Wallenberg syndrome) and cerebellar infarctions are the most common types of strokes. Occasionally, spinal cord infarctions occur because of the involvement of branches of the extracranial vertebral artery that supply the cervical spinal cord.

Intracranial vertebral artery dissections are more common in men. More than $50 \%$ of intracranial vertebral artery dissections are associated with subarachnoid haemorrhage. ${ }^{37}$ Brain stem infarctions and aneurismal arteries presenting as space occupying lesions are other manifestations. Basilar artery dissections are very rare.

\section{DIAGNOSIS OF CAD}

A high index of clinical suspicion is required to diagnose $C A D$ and confirmation is obtained by either Doppler ultrasonography, MRI/MRA, CT angiography, or catheter angiography.

Doppler ultrasound scanning has a diagnostic sensitivity of $>90 \%$. Continuous wave Doppler velocimetry, standard duplex scanning, and colour Doppler flow imaging have all been used in the diagnosis of carotid dissections. The common finding is that of a high resistance flow pattern in the distal arteries. Although this finding is not specific on its own, in combination with a characteristic clinical presentation and in absence of atherosclerosis it is highly suggestive of CAD. ${ }^{38}$ The pathognomonic feature of dissection, such as intramural haematoma or double lumen and intimal flap are rarely found. Transcranial Doppler can be used in diagnosis of the intracranial dissections. Doppler scanning is non-invasive, comparatively inexpensive, and is widely available. It can also be used for follow up monitoring of dissections. However, it has limitations including technical difficulties scanning the distal internal carotid artery, detecting emboli, and a lower sensitivity with dissections that cause low grade stenoses. In a study of 44 angiography confirmed dissections, continuous wave Doppler examination showed signs of severe obstruction of carotid arteries in $96 \%$ of cases (occlusion, extensive submandibular tight stenoses, significant slow down in the carotid and ophthalmic vessels, retrograde ophthalmic blood flow), standard duplex scanning suggested dissection in $72 \%$ of the cases (tapering stenoses or occlusion, segmental ectasis, tubular vessel, peripheral residual channel, or rare irregular "membrane") and colour Doppler flow imaging suggested a dissection in $82 \%$ of the cases. $^{38}$ The ultrasound failed only when it was done late and when moderate or segmental intrapetrosal dissections were present.

MRI/MRA is proving useful in diagnosing CAD. Intramural haematomas can be shown as hyperdense signals on $\mathrm{Tl}$ weighted imaging and characteristically have a crescent shape adjacent to the lumen. Fat suppression techniques are important to differentiate small intramural haematomas from the surrounding soft tissues. The MR scans can also show a luminal stenosis or an occlusion. With increasing experience in their use, MRI/MRA seem to be replacing catheter angiography in the diagnosis of CAD. However, the sensitivity of MRI/MRA is highest two days after dissections. MR imaging can also be used for follow up monitoring of the dissections.

CT angiography has been shown to have a very high sensitivity for CAD, but there is only limited experience with the technique. Unlike the other modalities, changes are apparent very soon after ictus.

Catheter angiography has been the traditional method of diagnosing arterial dissections. The commonest finding on angiography is the so called "string sign" - a long segment of narrowed lumen. The pathognomonic features of dissection, such as an intimal flap or a double lumen, are found in less than $10 \%$ of cases. $^{39}$ The artery may show sudden tapering because of occlusion of the lumen (fig 2), aneurysmal dilatations are also found in some cases. ${ }^{40}$ Catheter angiography is an invasive test and carries a risk of stroke of between $0.5 \%$ and $1 \% .{ }^{41}$ It is losing its "gold standard" status as the resolution of MR angiography now approaches that of a conventional angiography, and MRI can show the intramural haematoma itself.

\section{TREATMENT OF CAD}

Asymptomatic CADs do not require any intervention. The treatment of symptomatic extracranial dissections is

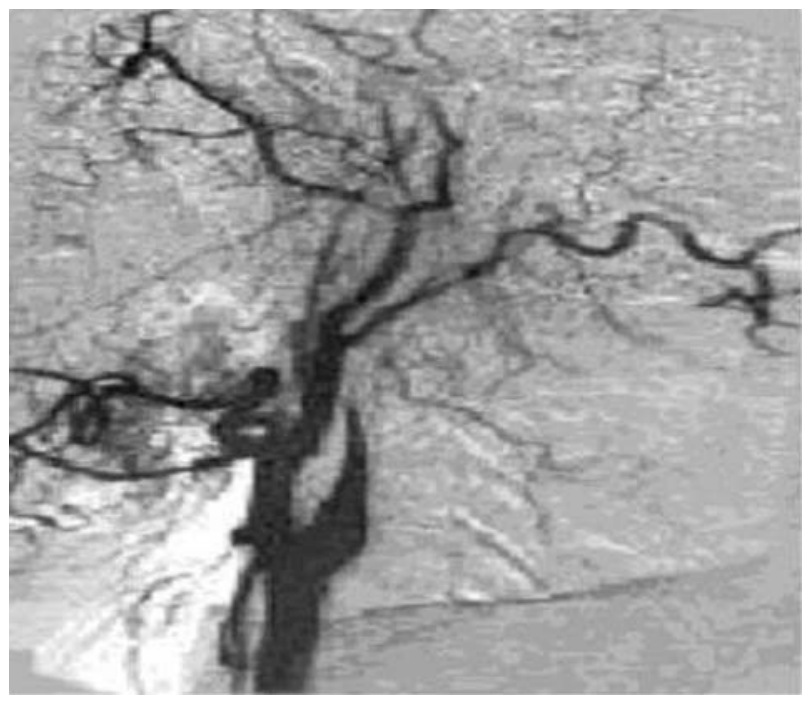

Figure 2 Carotid angiogram showing tapering of the blood flow in the left internal carotid artery because of the dissection. 
essentially medical. The rationale of treatment in CAD is to prevent thrombus formation on the injured endothelial surface and avoid artery to artery embolism. ${ }^{42}$ Once a complete occlusion has occurred, the artery does not recanalise. Anticoagulants and antiplatelet agents are commonly used in the treatment of CADs. A recent Cochrane review of the use of antithrombotic drugs (antiplatelet drugs, anticoagulants) in the treatment of extracranial carotid dissections concluded that there were no randomised trials comparing either anti-

coagulants or antiplatelet drugs with control. ${ }^{43}$ There is, therefore, no evidence to support their routine use in the treatment of extracranial internal carotid artery dissection, nor have the reported non-randomised studies shown any evidence of a significant difference between the anticoagulants therapy and antiplatelets.

Based on some open label studies and personal experience, several authorities favour anticoagulation with heparin followed by warfarin therapy as a reasonable approach in symptomatic CADs. ${ }^{42-46}$ In a recent one year follow up study, patients who were treated with aspirin had higher event rates (TIAs, stroke, or death) compared with patients treated with anticoagulants (12.4\% compared with $8.3 \%){ }^{47}$ However, the difference was statistically non-significant. There are no data from controlled studies on the safety of heparin, although several consecutive series showed no significant side effects ${ }^{48}{ }^{49}$ and transcranial Doppler monitoring showed decreased frequencies of high intensity transient signals with heparin treatment. ${ }^{50}$ In a study of the treatment of a blunt carotid artery injury, anticoagulation was found to be the treatment of choice where dissection or pseudoaneurysm was diagnosed. ${ }^{51}$ When anticoagulation therapy is used, it is continued until the luminal stenosis improves. After an initial period of a few weeks of treatment, anticoagulants may be substituted with antiplatelet agents (for example, aspirin, clopidogrel), and these can also be used as an initial treatment in patients with contraindications to anticoagulation. Several studies have shown the usefulness of the Doppler ultrasound in follow up monitoring of CADs. ${ }^{48} 52$ There is certainly an urgent need for a large randomised controlled trial comparing the efficacy and safety of anticoagulants and antiplatelets in CAD.

\section{SURGICAL TREATMENT OF CAD}

An urgent surgical intervention may be required in patients presenting with subarachnoid haemorrhage. Symptomatic aneurismal dilatation of the artery may also warrant surgery. Chronic carotid dissections have also been treated by surgical reconstruction to prevent further ischaemic or thromboembolic complications, if medical treatment with six month anticoagulation fails or if carotid aneurysms and/or high grade stenosis persist. ${ }^{53}$ Surgical interventions include endovascular treatment and the arterial repair.

\section{Prognosis}

Extracranial CADs generally carry a good prognosis. A literature review reports $50 \%$ of cases having no neurological deficit, $21 \%$ mild deficits only, and $25 \%$ moderate to severe deficits, the remaining $4 \%$ having died. ${ }^{54}$ In a recent Italian study looking at the relation between recanalisation rate and neurological outcome, no relation was found between these two variables, ${ }^{55}$ the neurological outcome was dependent on the lesion localisation and the presence of good collaterals.

Intracranial dissections are usually associated with severe neurological deficits or subarachnoid bleed and carry a poor prognosis. The recurrence rate for CAD is usually low. Higher recurrence rates have been noted in the immediate postdissection period and CAD associated with familial disorders of connective tissue. There is no evidence to suggest that anticoagulation or antiplatelet therapy prevents recurrence of CAD.

\section{Authors' affiliations}

B Thanvi, Department of Integrated Medicine, Leicester General Hospital, University Hospitals of Leicester NHS Trust, Leicester, UK S K Munshi, S L Dawson, Department of Stroke Medicine, Leicester General Hospital

T G Robinson, Leicester Warwick Medical School, Leicester General Hospital

Funding: none.

Conflicts of interest: none declared.

\section{REFERENCES}

1 Fisher CM, Ojemann RG, Roberson GH. Spontaneous dissection of cervicocerebral arteries. Can J Neurol Sci 1978;5:9-19.

2 Schievink WI, Mokri B, O'Fallon WM. Recurrent spontaneous cervical-artery dissection. N Engl J Med 1994;330:393-7.

3 Bogousslavsky J, Pierre P. Ischaemic stroke in patients under age 45. Neurol Clin 1992:10:113-24

4 Schievink WI, Mokri B, Whisnant JP. Internal carotid artery dissection in a community: Rochester, Minnesota, 1987-1992. Stroke 1993;24:1678-80.

5 Caplan LR, Tettenborn B. Vertebraobasilar occlusive disease: review of selected aspects: I. Spontaneous dissection of extracranial and intracranial posterior circulation arteries. Cerebrovasc Dis 1992;2:256-65.

6 Brandt T, Orbek E, Hacke W. Cervical artery dissection syndromes. In: Bogousslavsky J, Caplan L, eds. Stroke syndromes. 2nd ed. Cambridge: Cambridge University Press, 2001:660-6.

7 Schievink W. I. Spontaneous dissection of the carotid and vertebral arteries. N Engl J Med 2001;344:898-906.

8 Pepin M, Schwarze U, Superti-Furga A, et al. Clinical and genetic features of Ehlers-Danlos syndrome type IV, the vascular type. N Engl J Med 2000;342:673-80.

9 Brandt T, Hausser I, Orberk E, et al. Ultrastructural connective tissue abnormalities in patients with spontaneous cervicocerebral artery dissections. Ann Neurol 1998;44:281-5.

10 Chabriat H, Tournier-Laserve E, Bousser MG. Vasculopathies. In: Alberts M, ed. Genetics of cerebrovascular disease. New York: Futura, 1999

11 Dzrewas R, Konrad C, Drager B, et al. Cervical artery dissections-clinical features, risk factors, therapy and outcome in 126 patients. J Neurol 2003;250:1179-84.

12 Vila $N$, Millan $M$, Ferrer $X$, et al. Levels of $\alpha$ l-antitrypsin in plasma and risk of spontaneous cervical artery dissection: a case control study. Stroke 2003;34:E168-9.

13 Schievink WI, Thompson RC, Yong WH. Asyndrome of spontaneous cerebral and cervical artery dissection with angiolipomatosis. Report of two cases. J Neurosurg 2003;98:1124-7.

14 Schievink WI, Michels W, Mokri B, et al. A familial syndrome of arterial dissections with lentiginosis. N Engl J Med 1995;332:576-9.

15 Schievink WI, Mokri B, Piepgras DG, et al. Recurrent spontaneous arterial dissections: risk in familial versus nonfamilial disease. Stroke 1996;27:622-4.

16 Kuivaniemi H, Prockop DJ, Wu Y, et al. Exclusion of mutations in the gene for type III collagen (COL3A1) as a common cause of intracranial aneurysms or cervical artery dissections: results from sequence analysis of the coding sequences of type III collagen from 55 unrelated patients. Neurology 1993;43:2652-8

17 Rouviere S, Michelini R, Sarda P, et al. Spontaneous carotid artery dissection in two siblings with osteogenesis imperfecta. Cerebrovasc Dis 2004; 17:270-2.

18 Gallai V, Caso V, Paciaroni M, et al. Mild hyperhomocyst (e) inemia: a possible risk factor for cervical artery dissection. Stroke 2001;32:714-18.

19 Pezzini A, Del Zotto E, Archetti S, et al. Plasma homocysteine concentration C677T MTHFR genotype, and 844ins68bp CBS genotype in young adults with spontaneous cervical artery dissection and atherothrombic stroke. Stroke 2002;33:664-9.

20 Rosner AL. Spontaneous cervical artery dissections and implications for homocysteine. J Manipulative Physiol Ther 2004;27:124-32.

21 Schievink WI, Mokri B, Piepgras DG. Angiographic frequency of saccular intracranial aneurysms in patients with spontaneous cervical artery dissection. J Neurosurg 1992;76:62-6.

22 Barbour PJ, Castaldo JE, Rae-Grant AD, et al. Internal carotid artery redundancy is significantly associated with dissection. Stroke 1994;25:1201-6.

23 Lucas C, Lecroart JL, Gautier C, et al. Impairment of endothelial function in patients with spontaneous cervical artery dissection: evidence for a general arterial wall disease. Cerebrovasc Dis 2004;17:170-4.

24 Grau AJ, Brandt T, Buggle $F$, et al. Association of cervical artery dissection with recent infection. Arch Neurol 1999;56:851-6.

25 Guillon B, Berthet K, Benslamia L, et al. Infection and the risk of spontaneous cervical artery dissection: a case-control study. Stroke 2003;34:e79-81.

26 Schievink WI, Wiidicks EFM, Kuiper JD. Seasonal pattern of spontaneous cervical artery dissection. J Neurosurg 1998;89:101-3.

27 D'Anglejan-Chatillon J, Ribeiro V, Mas JL, et al. Migraine-a risk factor for dissection of cervical arteries. Headache 1989;29:560-1. 
28 Benninger DH, Georgiadia D, Kremer C. Mechanism of ischaemic infarction in spontaneous carotid dissection. Stroke 2004;35:482-5.

29 Bogousslavsky J, Despland PA, Regli F. Spontaneous carotid dissection with acute stroke. Arch Neurol 1987;44:137.

30 Silbert PL, Mokri B, Schievink WI. Headache and neck pain in spontaneous internal carotid and vertebral artery dissections. Neurology 1995;45:1517-22.

31 Biousse VD, Anglejan-Chatillon J, Massion H, et al. Head pain in nontraumatic carotid artery dissection: a series of 65 patients. Cephalagia 1994; 14:33-6.

32 Hess DC, Sethi KD, Nichols FT. Carotid dissection: a new false localizing sign. J Neurol Neurosurg Psychiatry 1990;53:804-5.

33 Guillon B, Levy C, Bousser MG. J Neurol Sci 1998;153:146-58.

34 Biousse V, Schaison M, Touboul P-J, et al. Ischaemic optic neuropathy associated with internal carotid artery dissection. Arch Neurol 1998;55:715-19.

35 Hart RG, Easton JD. Dissections. Stroke 1985;16:925-7.

36 Hinse $\mathbf{P}$, Thie A, Lachenmayer $L$. dissection of the extracranial vertebral artery: report of four cases and review of the literature. J Neurol Neurosurg Psychiatry 1991;54:863

37 Anonymous. Subarachnoid haemorrhage from intracranial dissecting aneurysm. J Neurosurg 1984;60:325

38 Steinke W, Rautenberg W, Schwartz A. Non invasive monitoring of internal carotid artery dissections. Stroke 1994;25:998-1005.

39 Houser OW, Mokri B, Sundt TM Jr, et al. Spontaneous cervical cephalic arterial dissection and its residuum: angiographic spectrum. AJNR Am J Neuroradiol 1984;5:27-34.

40 Diouhri H, Guillon B, Brunereau L, et al. MR angiography for the long-term follow-up of dissecting aneurysms of the extracranial internal carotid artery. AJR Am J Roentgenol 2000;174:1137-40.

41 Johnston DCC, Chapman KM, Goldstein LB. Low rate of complications of cerebral angiography in routine clinical practice. Neurology 2001;56:1009-15.
42 Stapf C, Elkind MSV, Mohr JP. Carotid artery dissection. Annu Rev Med 2000;51:329-47.

43 Lyrer $\mathrm{P}$, Engelter S. Antithrombotic drugs for carotid artery dissection. Stroke 2004;35:613.

44 Bassi P, Lattuado P, Gomitoni A. Cervical artery dissections: a multicentric prospective study (preliminary report). Neurol Sci 2003;24(suppl 1):S4-7.

45 Neau JP, Petit E, Gil R. Dissection of cervical arteries. Presse Med 2001;30:1882-9.

46 Selim M, Caplan LR. Carotid artery dissection. Curr Treat Options Cardiovasc Med 2004;6:249-53.

47 Beletsky V, Nadareishvili Z, Lynch J, et al. Cervical artery dissections: time for therapeutic trial? Stroke 2003;34:2856-60.

48 Sturzenegger $\mathbf{M}$. Spontaneous internal carotid artery dissection: early diagnosis and management in 44 patients. J Neurol 1995;242:231-8.

49 Desfontaines P, Despland PA. Dissection of internal carotid artery: aetiology, symptomatology, clinical and neurosonological follow-up and treatment in 60 consecutive cases. Acta Neurol Belg 1995;95:226-34.

50 Srinivasan J, Newell DW, Sturzenegger M, et al. Transcranial Doppler in the evaluation of internal carotid artery dissection. Stroke 1996;27:1226-30.

51 Singh RR, Barry MC, Ireland A, et al. Current diagnosis and management of blunt internal carotid artery injury. Eur J Vasc Endovasc Surg 2004;27:577-84

52 Sturzenegger $\mathbf{M}$, Mattle HP, Rivoir A, et al. Ultrasound findings in carotid artery dissection: analysis of 43 patients. Neurology 1995:45:691-8.

53 Muller BT, Luther B, Hort W, et al. Surgical treatment of 50 carotid dissections: indications and results. J Vasc Surg 2000;31:980-8.

54 Saver JL, Easton JD. Dissection of cervicocerebral arteries. In: Barnett HJM, Mohr JP, Stein BM, et al, eds. Stroke: pathophysiology, diagnosis and management. New York: Churchill Livingstone, 1998:769-86, 1459.

55 Caso V, Paciaroni M, Corea F, et al. Recanalization of cervical artery dissection: nfluencing factors and rate in neurological outcome. Cerebrovasc Dis 2004; 17:93-7.

\section{Call for papers}

11 th European Forum on Quality Improvement in Health Care 26-28 April 2006, Prague, Czech Republic

Deadline 30 September 2005.

For further information and to submit online go to: www.quality.bmipg.com 